\title{
Early Check in to Evaluate the Segmentation for Skin Lesions based on Modern Swarm Intelligence System.
}

\author{
Mohanad Aljanabi ${ }^{1 *}$, Jameel Kadhim Abed ${ }^{2}$, Ahmed R. Ajel ${ }^{3}$, Jabbar k. Mohammed ${ }^{4}$ \\ $\left\{{ }^{*}\right.$ Com.mhn@atu.edu.iq ${ }^{1}$, dr_jameel57@mtu.edu.iq ${ }^{2}$, dr_ahmed.R@mtu.edu.iq ${ }^{3}$,uotechnology.edu.iq@ 110214 $\}$ \\ ${ }^{1}$ Department of Electrical Power Techniques Engineering, Technical College /AL- Mausaib, Al-Furat Al- Awsat \\ Technical University Najaf, Iraq. \\ ${ }^{2}$ Department of Medical Instrumentation Techniques Engineering, Electrical Engineering Technical College, Middle \\ Technical University, Baghdad 1022, Iraq. \\ ${ }^{3}$ Department of Control and Automation Engineering, Electrical Engineering Technical College, Middle \\ Technical University, Baghdad 1022, Iraq \\ ${ }^{4}$ Department of Communication Engineering, University of Technology, Baghdad, Iraq.
}

\begin{abstract}
In recent years, the incidence of skin lesions has been one of the most rapidly increasing of all commonly occurring cancers. This deadliest form of melanoma must be detected early to be effectively treated. Because of the trouble and objectivity of human clarification, a significant research field has developed around the computerized examination of dermoscopy images. One reason to apply swarm intelligence systems is that an optimal solution can be advanced with a sensible computational application. This work introduces an artificial bee colony technique ( $\mathrm{ABC}$ ), distinctions, and applications. The planned $\mathrm{ABC}$ is a more suitable algorithm and one that requires smaller amounts of factors that need to be adjusted in comparison to other modern artificial swarm intelligence techniques (MASITs) for distinguishing unhealthy in skin tumor lesions. In these swarm's intelligence optimization algorithms have been positively executed for melanoma problems and provided extraordinary results guidance to better prediction and investigation of the skin cancer lesions. The experimental outcomes propose that the planned process proficient a developed accuracy associated to the ground truth (GT) used skin lesions' dermatology. So, we will be able to use these in a future study with different databases.
\end{abstract}

Keywords: - MASITs, ABC Algorithm, Skin Lesions, Image Segmentation, Levels of Melanomas.

\section{Introduction}

In the 1960s and 1970s, the clinical analysis of unhealthy moles was founded by indications such as itching or ulceration and bleeding. The existence of these norms at the time of performance was related to poor diagnosis as these symptoms only show at highly developed levels of skin tumor lesions $[1,2]$. The step includes detecting malignancy characteristics depending on popular analytical techniques. The ABCD rule of dermoscopy, the 7-point checklist, and the Menzies method. These methods depend on the existence of certain texture patterns. All these approaches share approximately ordinary ideas and agree for the choice of exact structures which can be completed with the assistance of computers. In detail, the ABCD rule, the Menzies method, and the 7-point checklist try to simplify the model study method by analyzing just a small sub-set of dermoscopic features and creating a recording scheme [3]. These methodologies supported clinicians with a suitable measurable basis, but it did not prove sufficiently effective for CDL. The decision threshold is correspondingly problematic; at this point, it has been fixed depending on some ages of clinical knowledge. Several researchers assert that these edges may make possible high rates of incorrect diagnoses [4]. One possible approach would be to systematically remove all pigmented lesions, but, since most injuries have many lesions, this is an impossible exercise and would be very costly [5]. The idea of the ABC method and its efficiency for MM identified is subsequently evaluated as made known in Fig. 1. [6,7]. 
Border

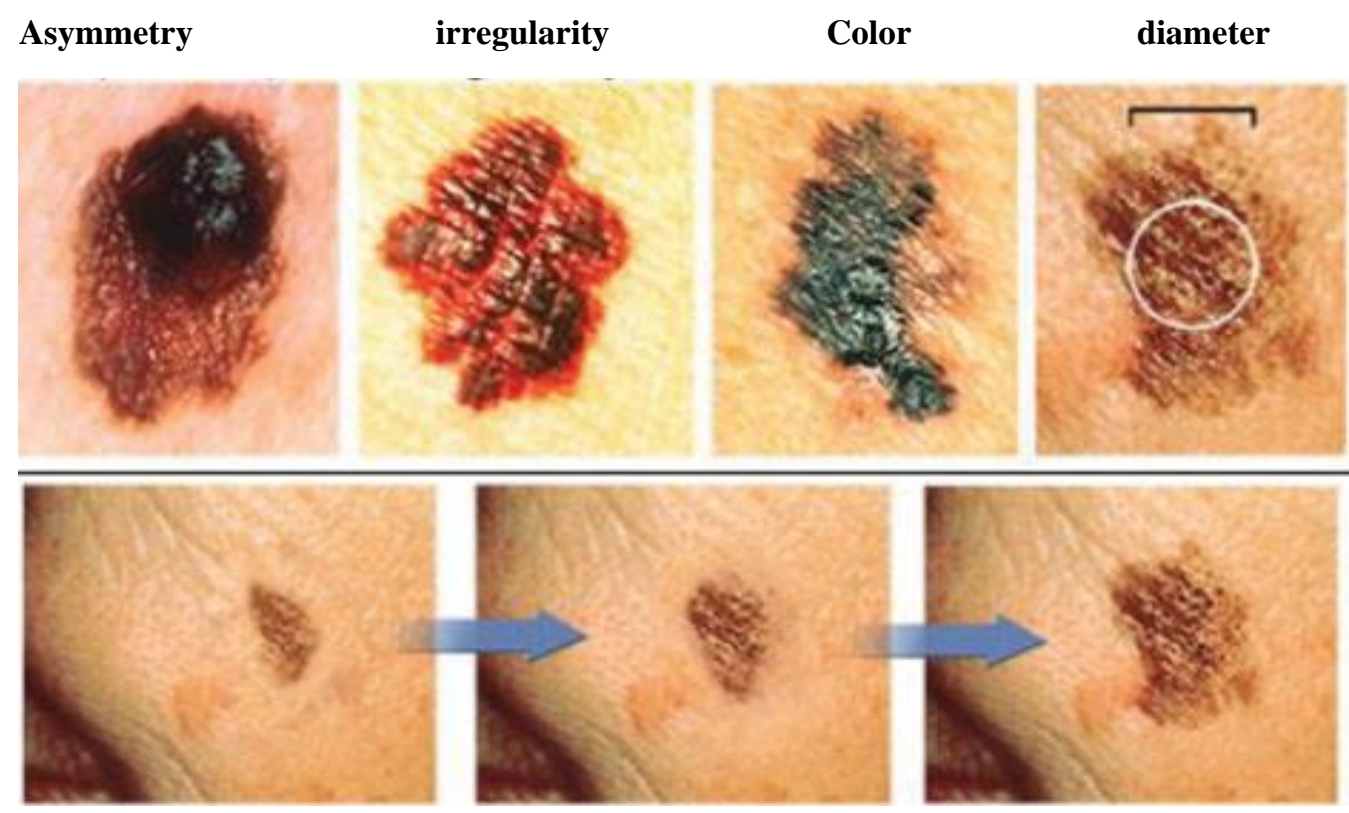

Evolution

Fig. 1. ABCD-E rule for Diagnosis of Melanoma.

In developed countries, the estimated five-year average survival rate is $73 \%$, and in developing countries, the median average survival rate is $56 \%$. The world average is estimated to be $69 \%$. While it accounts for only $4 \%$ of skin lesions, melanoma is the most serious because of its high possibility of metastatic [8]. In 2006, 2710 new cases in men and 3050 new cases in women were estimated, according to the estimates by Cancer Incidence in Brazil (CIB). In 2011, it is estimated that there is a sharp increase in the number of cancer cases, with the highest rates being found in the southern region, mainly in pale skin populations. The yearly rates of every form of skin cancer (tumor) are rising each one year, and skin cancer is one of the most widely spread forms of human cancer. At present, a total of about 3.5 million cases of skin cancers occur each year in the USA $[9,10]$. Melanomas (unhealthy nevi) are rapidly developing and greatly melanoma cancers frequently pervasion to nearby lymph nodes, and lungs as shown in Fig. 2. [5]. Fig. 3. Illustration of automatic analysis of healthy and unhealthy moles.

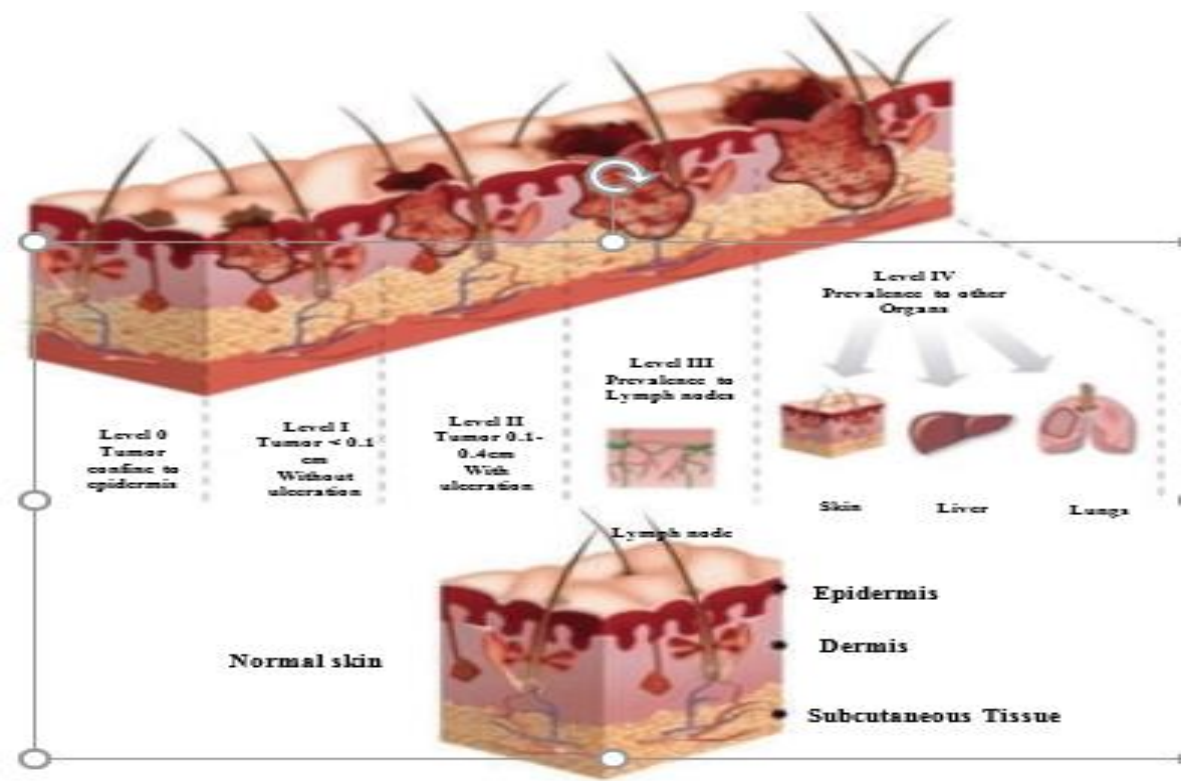

Fig. 2. Evaluation between healthy skin and skin artificial by MM performance of five levels in the unhealthy nevi development procedure. 
In Korotkov and Garcia (2012) [11] a literary review of the ways of detecting melanoma has also been made. However, this research considered only the best descriptors, along with the best forms of classification. In the data presented, a part of the literature uses the descriptors of Ham lick, Gabor, and to describe text, the borders, and statistics in color channels for coloring. The characteristics extracted by these descriptors can describe in detail the skin cancer, providing more accurate data for its identification. Barata et al. (2014) [12] two approaches of melanoma diagnosis are compared.

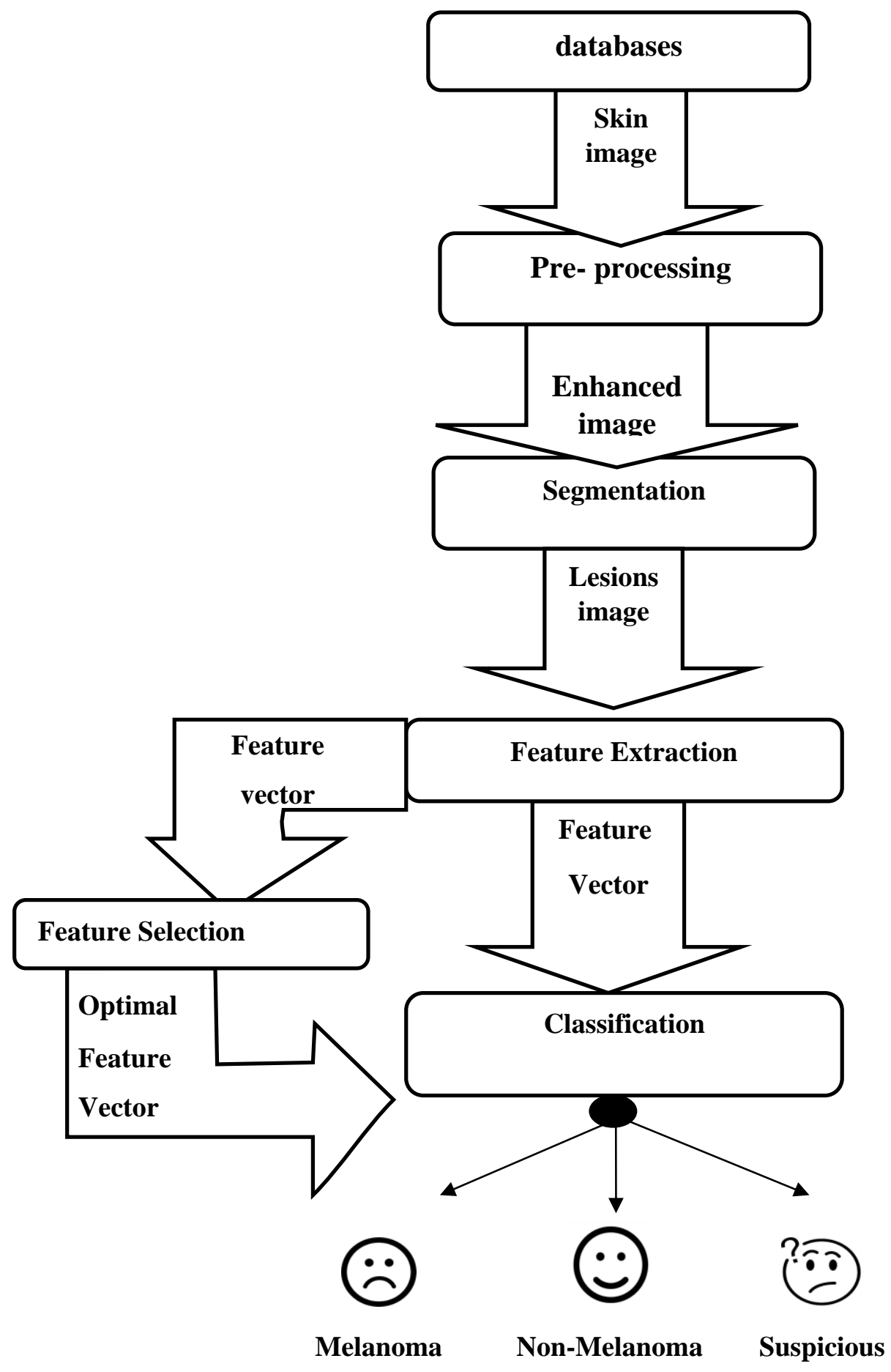

Fig.3. Representation illustration of automatic diagnosis of skin le 
The first approach, called "global", uses an adaptive threshold segment and an taking out of color and texture-based structures with histograms. The classification of these data was performed using A boost, SVM, and KNN algorithms. The second approach, called "local", identifies the best points of lesions through histograms. These data are classified using the Bag of Features (BOF) method. In this sense, the proposed approaches are tested with 200 dermatoscopic images and the results obtained with the global form reached a sensitivity of $93 \%$ and a specificity of $85 \%$ in the identification of MM, while the local form got a sensitivity of $96 \%$ and a specificity of $80 \%$ [13].

\section{Method}

It is well-identified that for the inhabitants-based algorithms the consideration and the exploitation capabilities are both required truths. The examination ability refers to the ability to investigate the different unknown areas to notice the global optimum in resolution space, while the exploitation ability discusses to the put into use the information of the previous good resolutions to find better explanations. Table 1 displays the benefits for utilizing the $\mathrm{ABC}$ technique, the information updated to guide the honey bees and the detection of new areas by dance strategy [14].

Table. 1. Benefits of $\mathrm{ABC}$ technique

\begin{tabular}{|l|l|}
\hline Usages & Descriptions \\
\hline Simplicity & Easy and straightforward to implement ways. \\
\hline Flexibility & Improve new algorithms and develop, and flexible enough to modify. \\
\hline Easy Designing & Having lower parameters, it is easy to design it. \\
\hline Simple of Combination & It can be easily mixed with another algorithm. \\
\hline Richness & $\begin{array}{l}\text { Obtaining an optimal solution is a good connection with the communication in } \\
\text { the behavior nature of the cells. }\end{array}$ \\
\hline Randomly Behavior & Based on foraging behavior of the hive \\
\hline Easy of analysis & Generated solution is analysis in behavior nature. \\
\hline
\end{tabular}

There are various types of dermoscopy, both digital and analog, that can provide a high resolution of the skin lesion to apply it to the other levels. Working with a digital dermoscopy is simpler for saving the information and using it for the next steps. In Fig. 4, we show the analog dermoscopy that can be attached to the camera to take a high-resolution photo, except a. Moreover, $\mathrm{f}$ and $\mathrm{h}$ also show digital dermoscopy.

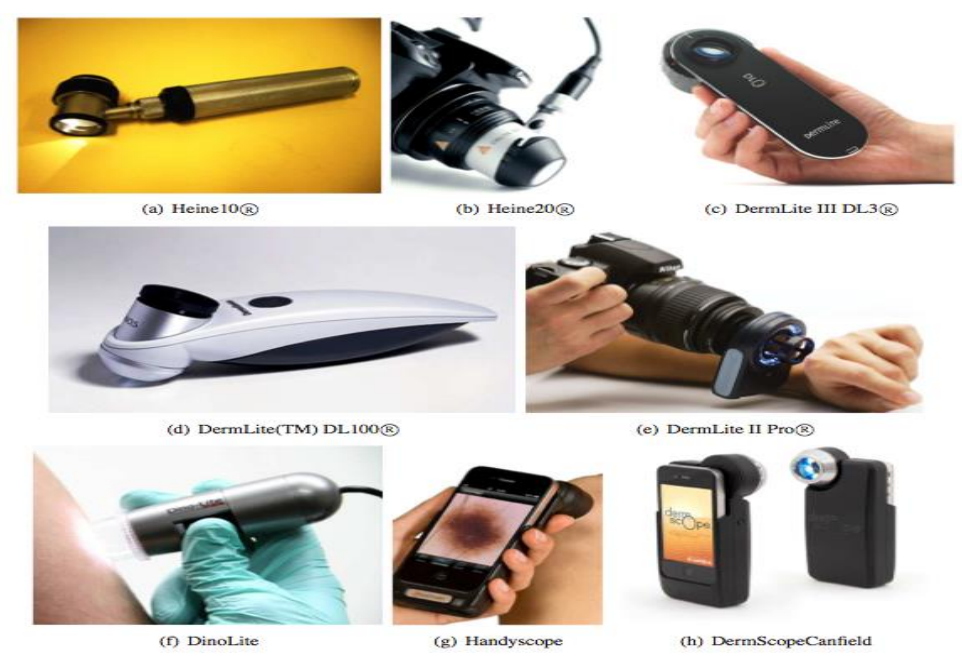


Fig. Error! No text of specified style in document.. ( a, b, c, d, and e) illustration are modern digital dermoscopy shown in $(f, g$, and $h)[15]$.

$\mathrm{D}, \mathrm{f}, \mathrm{g}$, and h show advanced digital dermoscopy, most of which can be attached to a mobile phone, and all the images can be sent to a specialist with high resolution [15]. One of the greatest difficulties in diagnosing skin tumors is the generating of an algorithm to diagnosis the border of the skin lesion automatically because each lesion has its structure; some of the challenges, shown in Fig. 5, are the presence of hair or oil, the bubble in the pictures, irregular borders, low contrast, or polychromatic color of the lesion, and segmentation[16]. Thus, in this study, we introduce various algorithms to diagnosis the lesion border automatically, and to have an accurate characteristic extraction for the following stages [17].

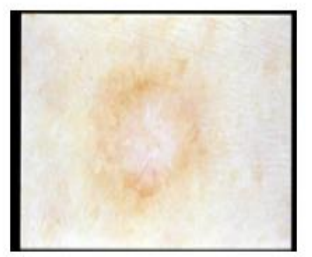

(a) Low contrast

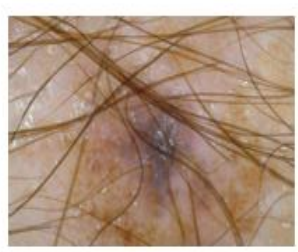

(e) Hairs

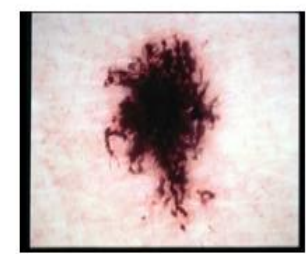

(b) Irregular border

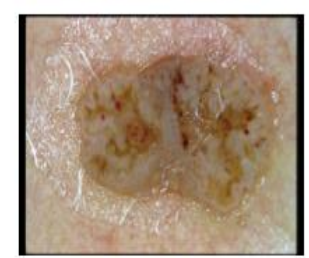

(f) Bubbles

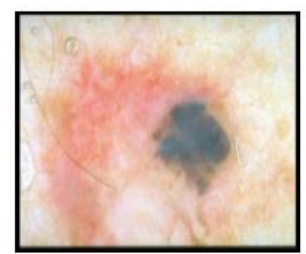

(c) Fuzzy border

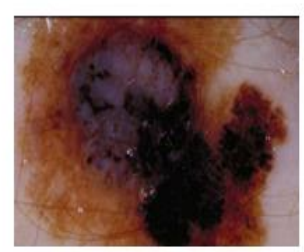

(g) Variegated coloring

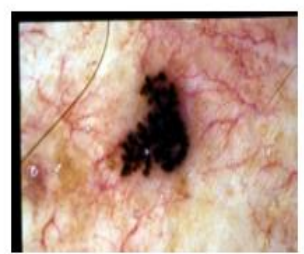

(d) Blood vessels

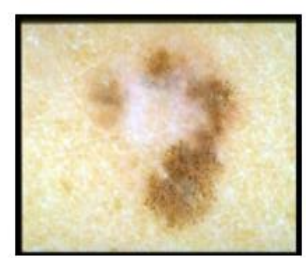

(h) Fragmentation

Fig.5. Problems with border detection[17].

\section{Results and discussion}

Wholly the approaches that stated above are good for diagnosing MM, but they also have approximately drawbacks that should be measured. First, it takes a lot of time to exploit all these algorithms and reasons for each lesion, so applying them to many different lesions is not a good method. Secondly, physicians in rural areas all over the world do not have access to dermatologists and specialists and lesions must be checked by physicians who do not have specialized knowledge of dermatology [18]. Additionally, there are various opinions and ideas about the calculation of the factors and have different approaches for distinguishing skin lesions. Because of all these difficulties, we want to research and create original automated procedures that are more elastic, faster, and more intelligent for diagnosing melanoma [19]. To make an automated algorithm for analyzing the data images of the skin lesion we have various steps. The flowchart for an automated MM detection scheme is shown in Fig.6 [20].

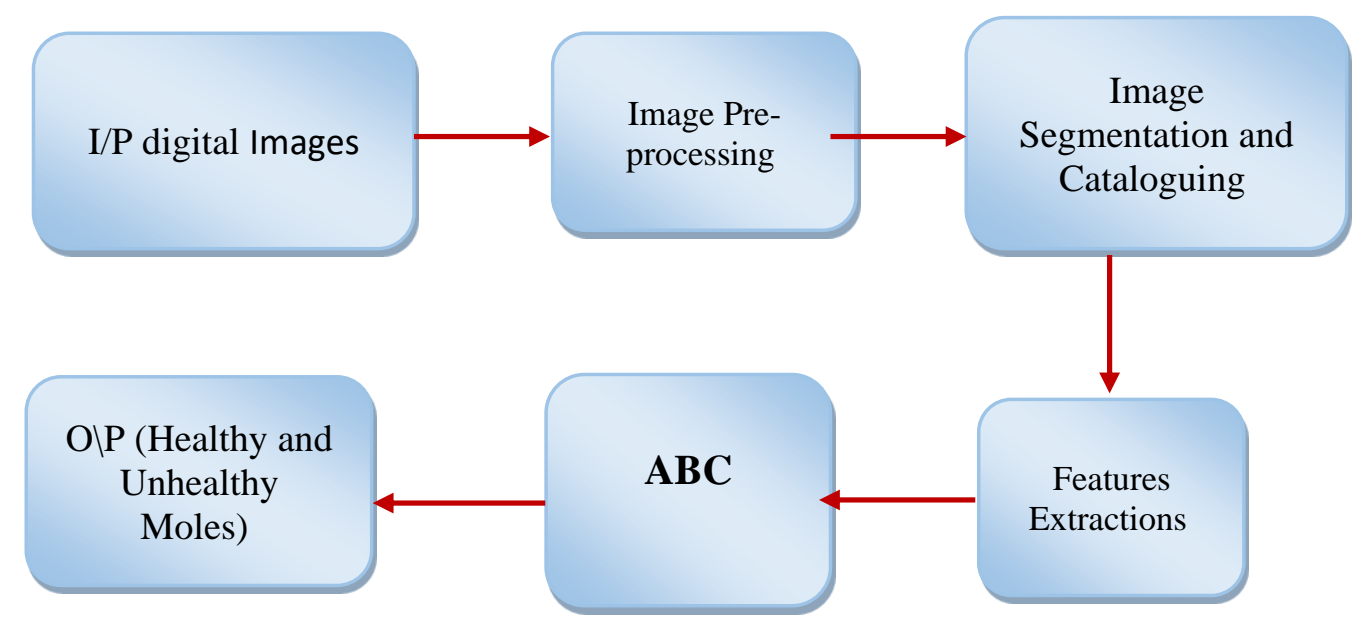


Fig.6. Block diagram illustration for an automated early healthy and unhealthy moles detection scheme [20].

Figure 7 demonstrates the outcomes found in the 4 data groups with images. The effectiveness of the planned style, when compared to the images, got by the methodology (green) of the model GT (white) [21]. Table 2. and Fig. 8. display an assessment between the planned technique and other approaches that applied ISBI 2017 data group in relationships of the accuracy (ACC), sensitivity (SEN), specificity (SPE), dice coefficient (DIC), and Jaccard index (JAC). Similarly, in Figure 8. and Table 2, the projected process reached the best-segmented outcome from the ISBI 2017 images. It can be experimental that the nearer superlativesegmented consequence was that of Guo et al. [26] concerning SEN, ACC, DIC, and JAC. For SPE, the outcomes of Li \& Shen [24] were nearby.

(1)

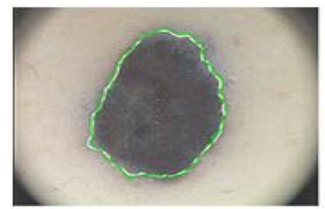

(2)

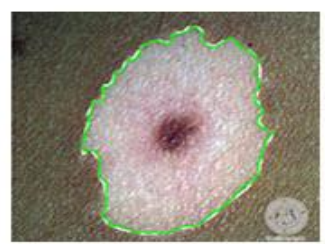

(3)

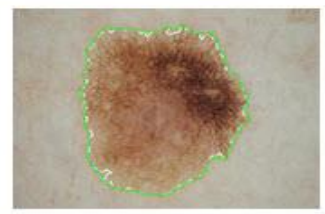

(4)

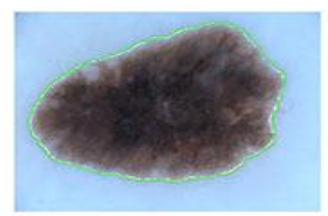

(a)
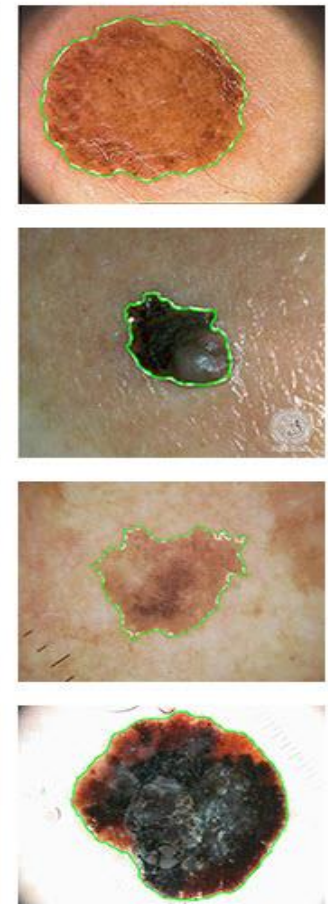

(b)
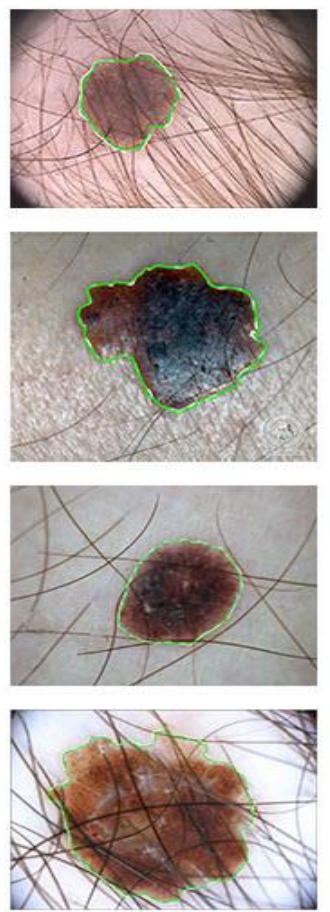

(c)
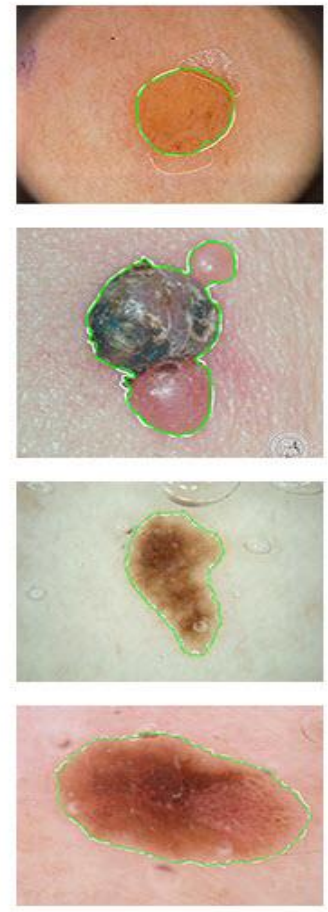

(d)

Fig. 7. Recognition outcomes for skin lesions: Row (1-4) PH2, ISBI (2016, 2017), DermIs data groups; Columns (a) various colored issue; (b) specular reflections.; (c) incidence of hair; and (d) the existence of bubbles.

Table 2. Comparisons of similar works with the projected style using the ABC algorithm for the ISBI 2017 data group.

\begin{tabular}{lccccc}
\hline Methods & SEN & SPE & ACC & DIC & JAC \\
\hline Proposed method & $\mathbf{9 8 . 6 4}$ & $\mathbf{9 8 . 5 2}$ & $\mathbf{9 7 . 6 1}$ & $\mathbf{9 2 . 3 1}$ & $\mathbf{8 4 . 2 6}$ \\
Yuan et al. [22] & 82.50 & 97.50 & 93.40 & 84.90 & 76.50 \\
Bi et al. [23] & 42.70 & 96.30 & 85.80 & 84.40 & 76.00 \\
Li \& Shen [24] & 82.00 & 97.80 & 93.20 & 84.70 & 76.20 \\
Al-masni et al. [25] & 85.40 & 96.69 & 94.03 & 87.08 & 77.11 \\
Guo et al. [26] & 97.50 & 88.80 & 95.30 & 90.38 & 80.32 \\
\hline
\end{tabular}




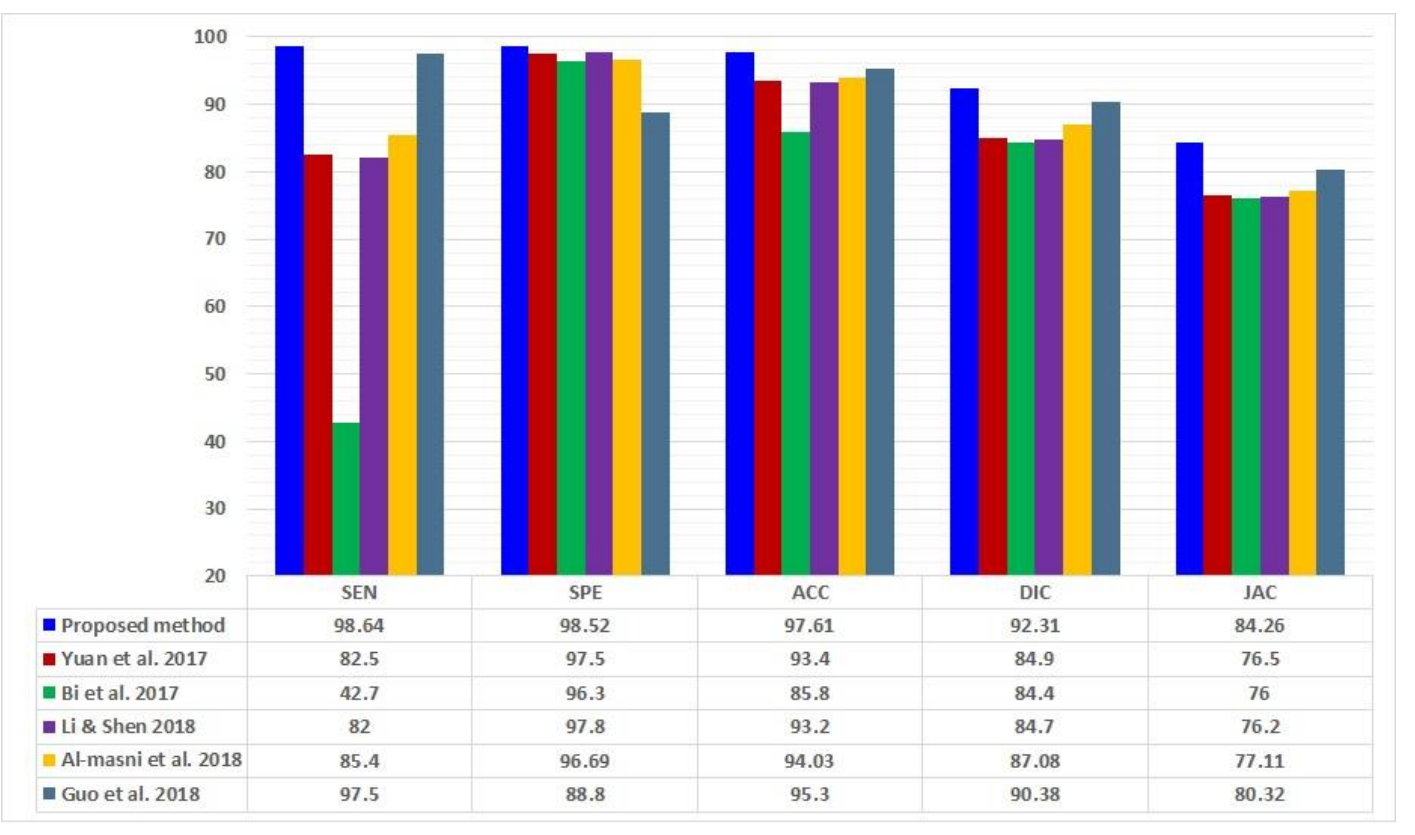

Fig. 8. The outcomes existing by the proposals based on the $\mathrm{ABC}$ algorithm compared to current procedures in the literature for the ISBI 2017 data groups.

\section{Conclusions}

The proposals suggested in this study accomplished good outcomes in the classification of melanoma. However, the form of recognition by the classification with $\mathrm{ABC}$ is still more advantageous concerning the recognition structure with trained models of the meta-heuristic method because we see better results for this configuration. The difference in results between approaches was explained by the number of images that were submitted in the experiments. The data augmentation operation applied to the datasets was not enough to mediate this problem; it requires a greater number of images and classes to produce better learning in the $\mathrm{ABC}$ architectures. Besides, we are planning to use metaheuristic methods that are original in the area, like the $\mathrm{ABC}$ algorithm, to enhance the accuracy of MM analysis. In future studies, the aim is to improve the recognition of melanoma by classification with $\mathrm{ABC}$ through the combination of new color spaces and new descriptors. This detection structure should still test other convolutional architectures to perform the classification of the obtained data. As future work, it is suggested that international bases be used to challenge the methodology proposed in the present research to develop techniques for the recognition of malignant and benign moles.

\section{References}

[1] W. Stolz, "ABCD rule of dermatoscopy: a new practical method for early recognition of malignant melanoma," Eur. J. Dermatol., vol. 4, pp. 521-527, 1994.

[2]H. Pehamberger, M. Binder, A. Steiner, and K. Wolff, "In vivo epiluminescence microscopy: improvement of early diagnosis of melanoma," Journal of Investigative Dermatology, vol. 100, no. 3, pp. S356-S362, 1993.

[3]G. Argenziano et al., "Dermoscopy of patients with multiple nevi: improved management recommendations using a comparative diagnostic approach," Archives of dermatology, vol. 147, no. 1, pp. 46-49, 2011.

[4]J. S. Henning et al., "The CASH (color, architecture, symmetry, and homogeneity) algorithm for dermoscopy," Journal of the American Academy of Dermatology, vol. 56, no. 1, pp. 45-52, 2007.

[5] R. Garnavi, M. Aldeen, M. E. Celebi, G. Varigos, and S. Finch, "Border detection in dermoscopy images using hybrid thresholding on optimized color channels," Computerized Medical Imaging and Graphics, vol. 35, no. 2, pp. 105-115, 2011.

[6]G. Argenziano, G. Fabbrocini, P. Carli, V. De Giorgi, E. Sammarco, and M. Delfino, "Epiluminescence microscopy for the diagnosis of doubtful melanocytic skin lesions: comparison of the ABCD rule of dermatoscopy and a new 7-point checklist based on pattern analysis," Archives of dermatology, vol. 134, no. 12, pp. 1563-1570, 1998.

[7]D. Gutman et al., "Skin lesion analysis toward melanoma detection: A challenge at the international symposium on biomedical imaging (ISBI) 2016, hosted by the international skin imaging collaboration (ISIC)," arXiv preprint arXiv:1605.01397, 2016. 
[8]M. Dowlatshahi and V. Derhami, "Winner Determination in Combinatorial Auctions using Hybrid Ant Colony Optimization and Multi-Neighborhood Local Search," Journal of AI and Data Mining, vol. 5, no. 2, pp. 169-181, 2017. [9]N. K. Mishra and M. E. Celebi, "An overview of melanoma detection in dermoscopy images using image processing and machine learning," arXiv preprint arXiv:1601.07843, 2016.

[10]N. Dey, V. Rajinikanth, A. S. Ashour, and J. M. R. Tavares, "Social group optimization supported segmentation and evaluation of skin melanoma images," Symmetry, vol. 10, no. 2, p. 51, 2018.

[11]K. Korotkov and R. Garcia, "Computerized analysis of pigmented skin lesions: a review," Artificial intelligence in medicine, vol. 56, no. 2, pp. 69-90, 2012.

[12]C. Barata, M. Ruela, M. Francisco, T. Mendonça, and J. S. Marques, "Two systems for the detection of melanomas in dermoscopy images using texture and color features," IEEE Systems Journal, vol. 8, no. 3, pp. 965-979, 2014.

[13]N. Codella, J. Cai, M. Abedini, R. Garnavi, A. Halpern, and J. R. Smith, "Deep learning, sparse coding, and SVM for melanoma recognition in dermoscopy images," in International Workshop on Machine Learning in Medical Imaging, 2015, pp. 118-126: Springer.

[14]F. M. Walter et al., "Using the 7-point checklist as a diagnostic aid for pigmented skin lesions in general practice: a diagnostic validation study," Br J Gen Pract, vol. 63, no. 610, pp. e345-e353, 2013.

[15]M. Sadeghi, "Towards prevention and early diagnosis of skin cancer: computer-aided analysis of dermoscopy images," Applied Science: School of Computing Science, 2018.

[16]Y. K. Jain and M. Jain, "Comparison between different classification methods with application to skin cancer," International Journal of Computer Applications, vol. 53, no. 11, 2012.

[17]K. Chatfield, K. Simonyan, A. Vedaldi, and A. Zisserman, "Return of the devil in the details: Delving deep into convolutional nets," arXiv preprint arXiv:1405.3531, 2014.

[18]J. A. Jaleel and S. Salim, "Implementation of ANN Classifier using MATLAB for Skin Cancer Detection," 2013.

[19]T. Mendonca et al., "Comparison of segmentation methods for automatic diagnosis of dermoscopy images," in Engineering in Medicine and Biology Society, 2007. EMBS 2007. 29th Annual International Conference of the IEEE, 2007, pp. 6572-6575: IEEE.

[20]Y. K. Jain and M. Jain, "Comparison between different classification methods with application to skin cancer," International Journal of Computer Applications, vol. 53, no. 11, 2012.

[21]K. Chatfield, K. Simonyan, A. Vedaldi, and A. Zisserman, "Return of the devil in the details: Delving deep into convolutional nets," arXiv preprint arXiv:1405.3531, 2017.

[22]Y. Yuan, M. Chao, and Y.-C. Lo, "Automatic skin lesion segmentation with fully convolutional-deconvolutional networks," arXiv preprint arXiv:1703.05165, 2017.

[23]L. Bi, J. Kim, E. Ahn, and D. Feng, "Automatic skin lesion analysis using large-scale dermoscopy images and deep residual networks," arXiv preprint arXiv:1703.04197, 2017.

[24]Y. Li and L. Shen, "Skin lesion analysis towards melanoma detection using deep learning network," Sensors, vol. 18 , no. 2, p. 556, 2018.

[25]M. A. Al-masni, M. A. Al-antari, M.-T. Choi, S.-M. Han, and T.-S. Kim, "Skin lesion segmentation in dermoscopy images via deep full resolution convolutional networks," Computer methods and programs in biomedicine, vol. 162, pp. 221-231, 2018.

[26]Y. Guo, A. S. Ashour, and F. Smarandache, "A Novel Skin Lesion Detection Approach Using Neutrosophic Clustering and Adaptive Region Growing in Dermoscopy Images," Symmetry, vol. 10, no. 4, p. 119, 2019. 